Supporting information

\title{
Perturbed Angular Correlation as a tool to study precursors for radiopharmaceuticals
}

Elena S. Kurakina ${ }^{1,2^{*}}$, Valery Radchenko ${ }^{3,4}$, Andrey N. Belozub ${ }^{1}$, Georgi Bonchev ${ }^{5}$, Gospodin A. Bozhikov $^{6}$, Atanas I. Velichkov ${ }^{7}$, Monika Stachura $^{3}$, Dimitar V. Karaivanov ${ }^{1,7}$, Eldar P. Magomedbekov $^{2}$, Dmitry V. Filosofov ${ }^{1}$

${ }^{1}$ Dzhelepov Laboratory of Nuclear Problems, Joint Institute for Nuclear Research, Dubna, 141980, Russian Federation

${ }^{2}$ Department of High-Energy Chemistry and Radioecology, D. Mendeleev University of Chemical Technology of Russia, Moscow, 125047, Russian Federation

${ }^{3}$ Life Sciences Division, TRIUMF, Vancouver, V6T 2A3, Canada

${ }^{4}$ Department of Chemistry, University of British Columbia, Vancouver, V6T 1Z1, Canada

${ }^{5}$ Military Medical Academy, 3, Chr. Smirnenski St., Varna 9010, Bulgaria

${ }^{6}$ Flerov Laboratory of Nuclear Reactions, Joint Institute for Nuclear Research, Dubna, 141980,

Russian Federation

${ }^{7}$ Institute for Nuclear Research and Nuclear Energy, Bulgarian Academy of Sciences, Sofia, BG1784, Bulgaria

*corresponding author: Kurakina E.S. kurakina@jinr.ru 


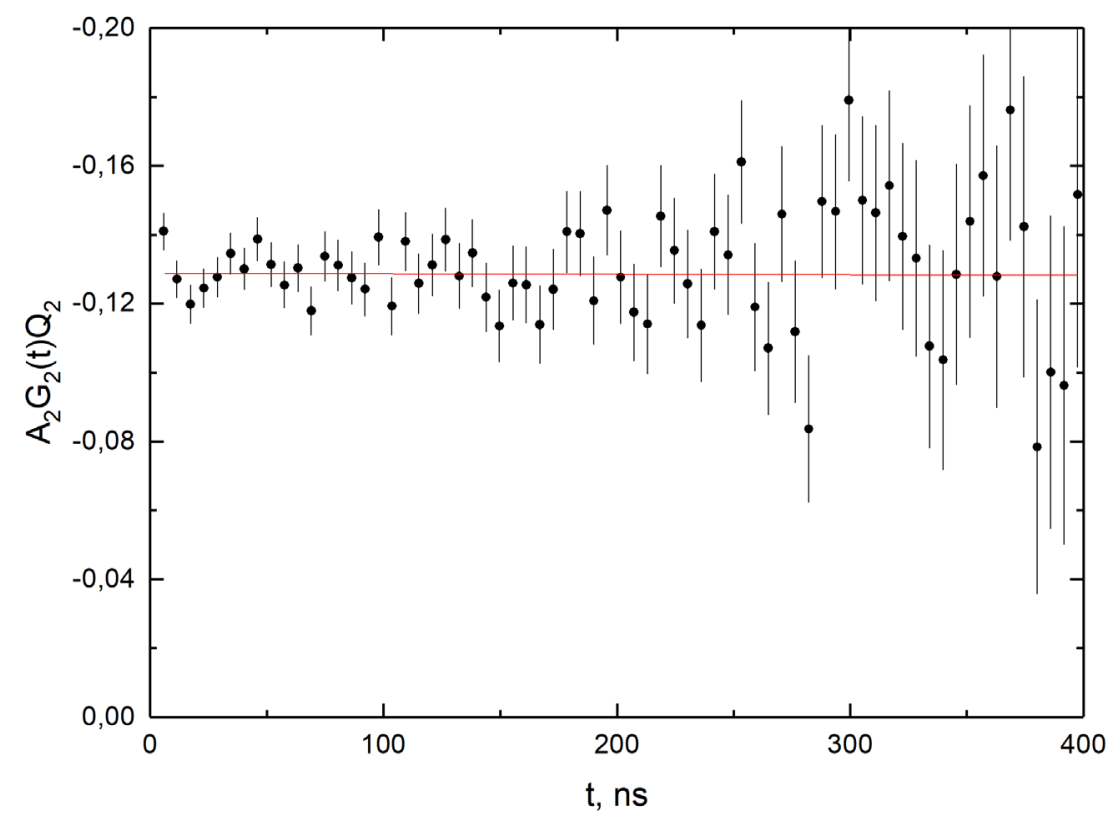

Figure S1. TDPAC spectra for ${ }^{111} \mathrm{In}$ at $\mathrm{C}(\mathrm{DTPA})=10^{-4} \mathrm{M}$, ionic strength $\mathrm{I}=0.5\left(\mathrm{HClO}_{4}\right.$, $\left.\mathrm{NaClO}_{4}, \mathrm{NaOH}\right)$ with $\mathrm{pH}=0.3$.

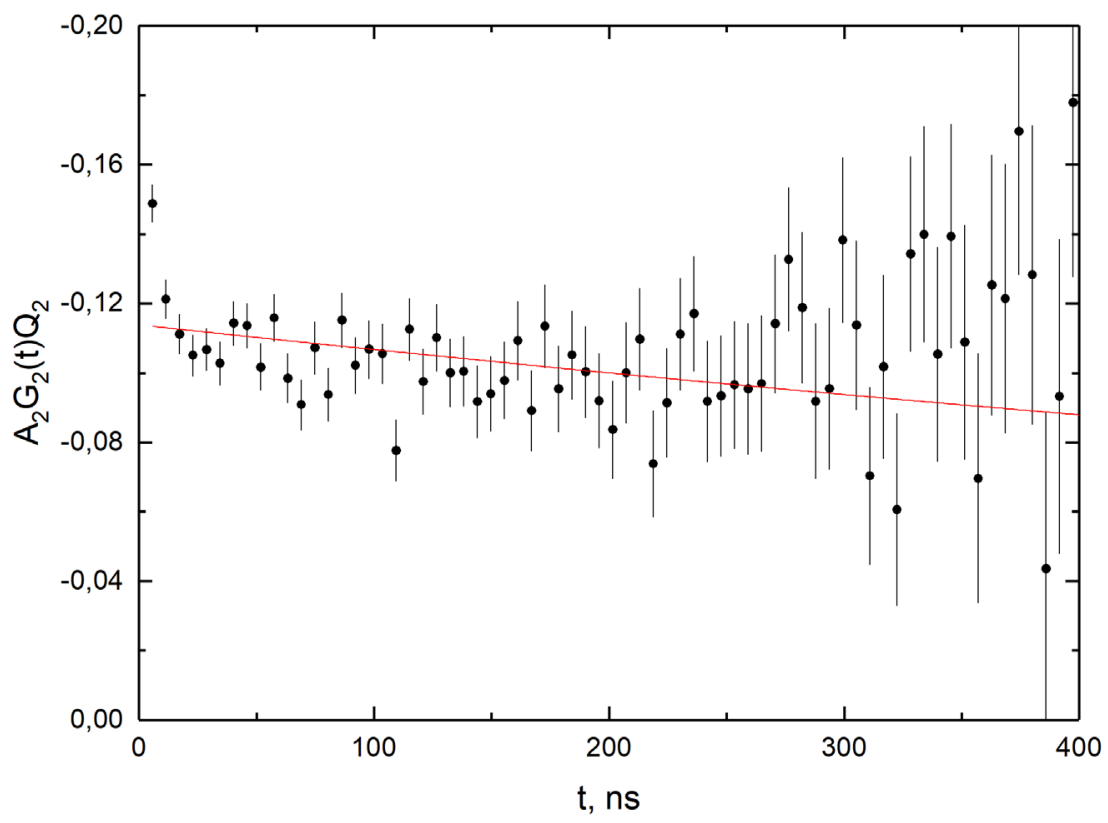

Figure S2. TDPAC spectra for ${ }^{111} \mathrm{In}$ at $\mathrm{C}(\mathrm{DTPA})=10^{-4} \mathrm{M}$, ionic strength $\mathrm{I}=0.5\left(\mathrm{HClO}_{4}\right.$, $\left.\mathrm{NaClO}_{4}, \mathrm{NaOH}\right)$ with $\mathrm{pH}=1.67$. 


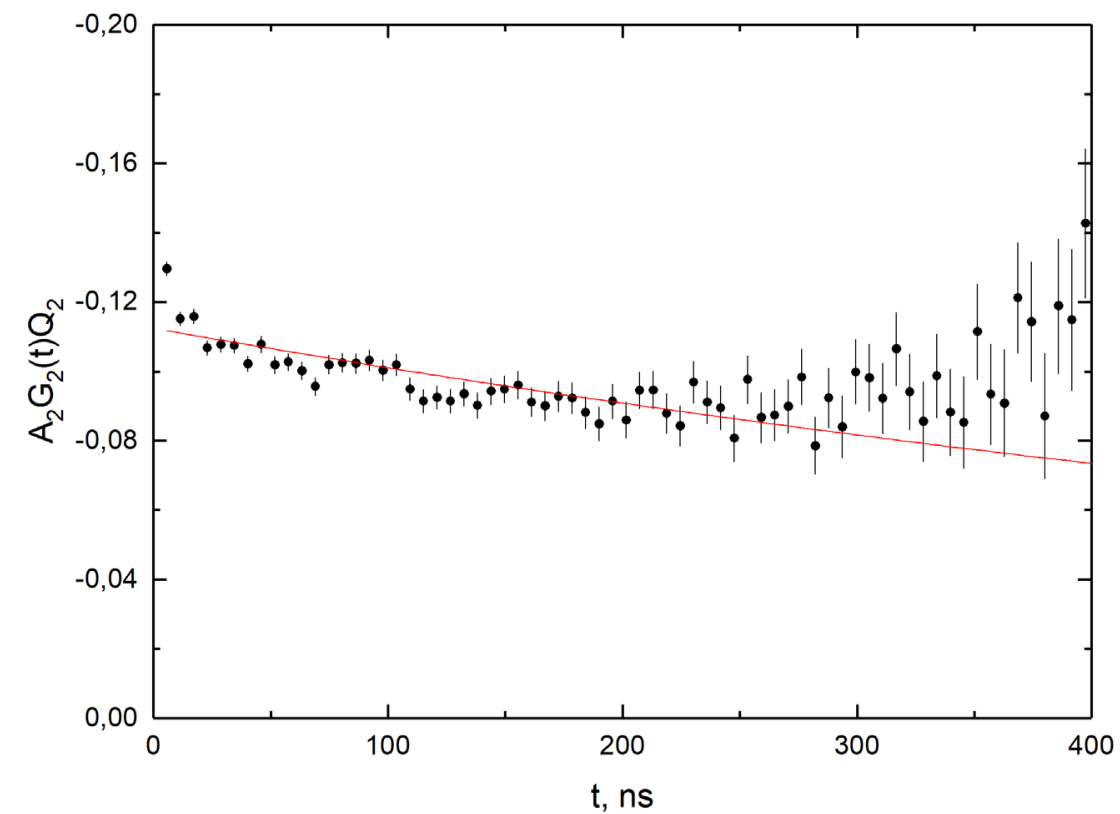

Figure S3. TDPAC spectra for ${ }^{111} \mathrm{In}$ at $\mathrm{C}(\mathrm{DTPA})=10^{-4} \mathrm{M}$, ionic strength $\mathrm{I}=0.5\left(\mathrm{HClO}_{4}\right.$, $\mathrm{NaClO}_{4}, \mathrm{NaOH}$ ) with $\mathrm{pH}=1.85$.

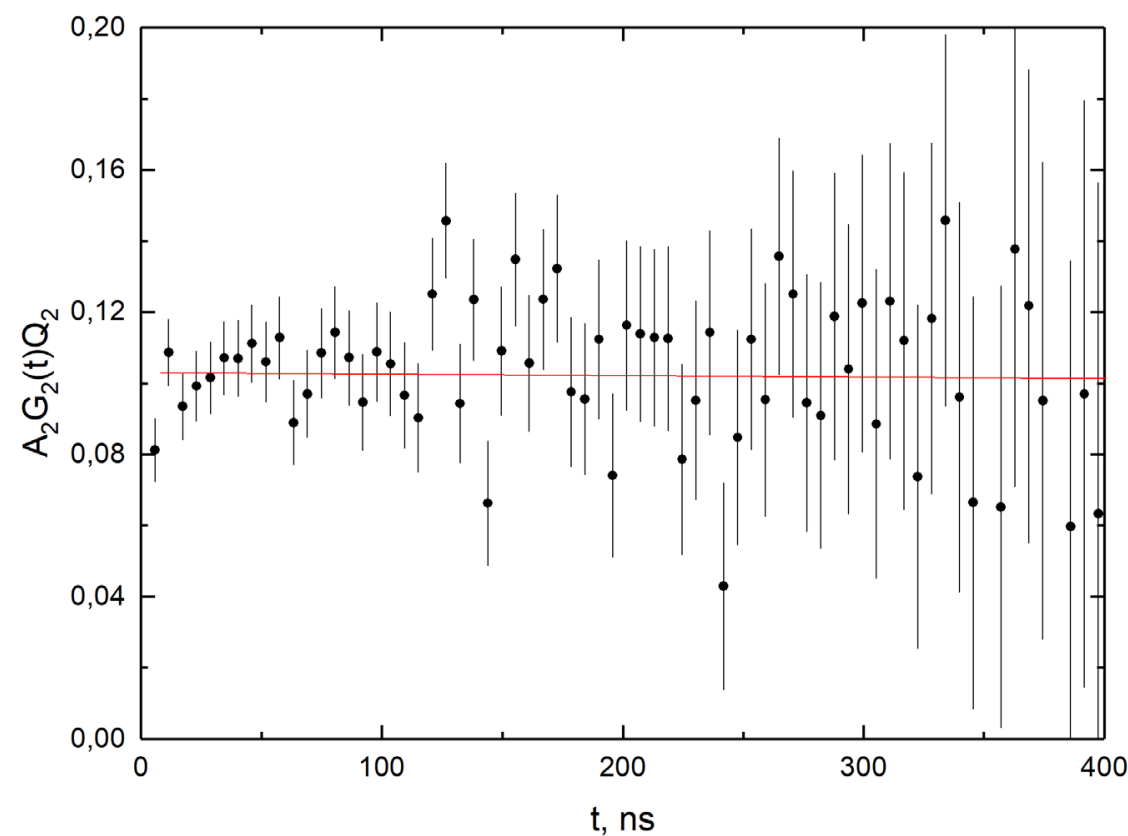

Figure S4. TDPAC spectra for ${ }^{111 \mathrm{~m}} \mathrm{Cd}$ at $\mathrm{C}(\mathrm{DTPA})=10^{-4} \mathrm{M}$, ionic strength $\mathrm{I}=0.5\left(\mathrm{HClO}_{4}\right.$, $\mathrm{NaClO}_{4}, \mathrm{NaOH}$ ) with $\mathrm{pH}=0.3$. 


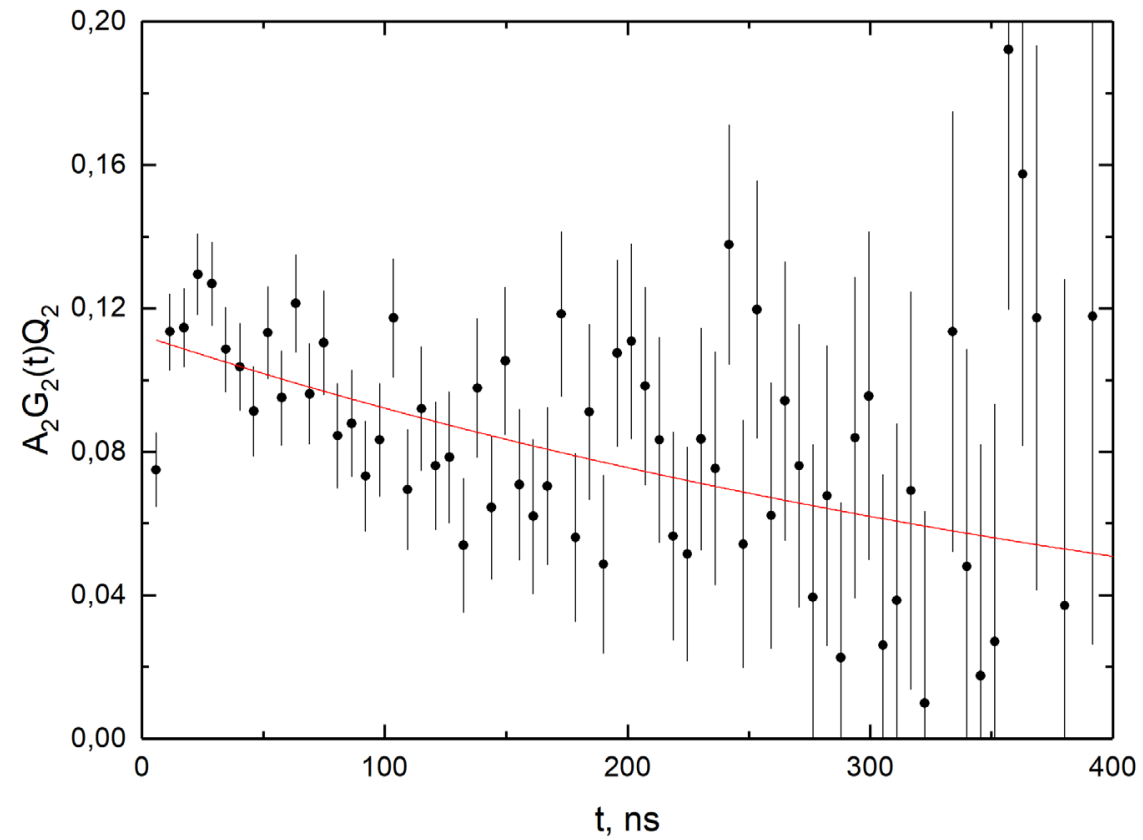

Figure S5. TDPAC spectra for ${ }^{111 \mathrm{~m}} \mathrm{Cd}$ at $\mathrm{C}(\mathrm{DTPA})=10^{-4} \mathrm{M}$, ionic strength $\mathrm{I}=0.5\left(\mathrm{HClO}_{4}\right.$, $\mathrm{NaClO}_{4}, \mathrm{NaOH}$ ) with $\mathrm{pH}=2$.

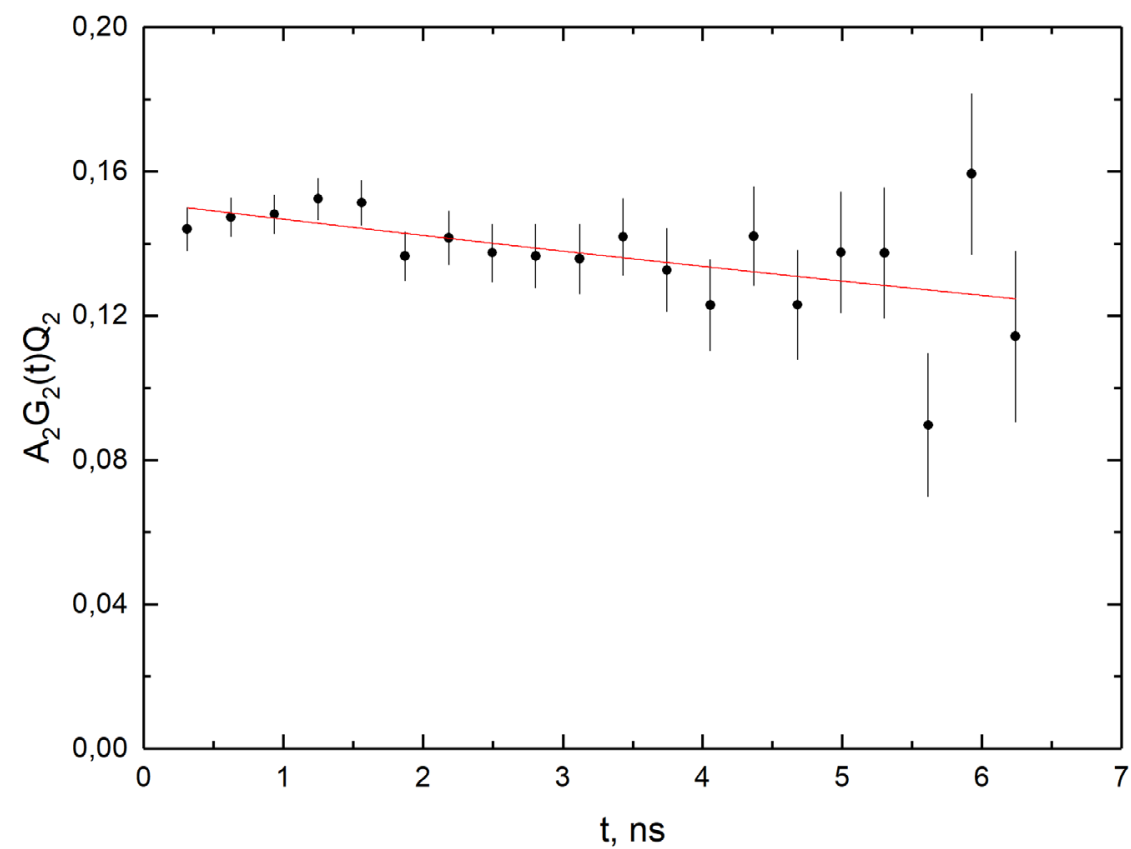

Figure S6. TDPAC spectra for ${ }^{154} \mathrm{Eu}$ at $\mathrm{C}(\mathrm{DTPA})=10^{-4} \mathrm{M}$, ionic strength $\mathrm{I}=0.5\left(\mathrm{HClO}_{4}\right.$, $\mathrm{NaClO}_{4}, \mathrm{NaOH}$ ) with $\mathrm{pH}=1$. 


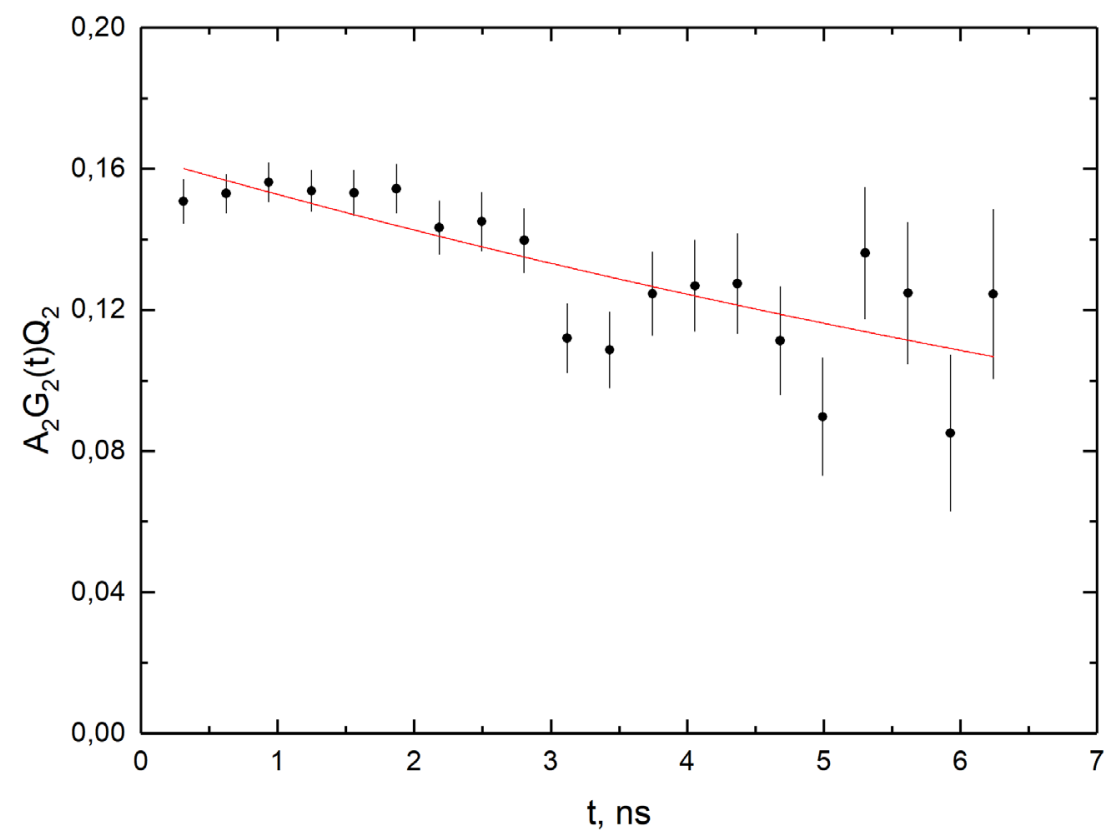

Figure S7. TDPAC spectra for ${ }^{154} \mathrm{Eu}$ at $\mathrm{C}(\mathrm{DTPA})=10^{-4} \mathrm{M}$, ionic strength $\mathrm{I}=0.5\left(\mathrm{HClO}_{4}\right.$, $\mathrm{NaClO}_{4}, \mathrm{NaOH}$ ) with $\mathrm{pH}=1.72$.

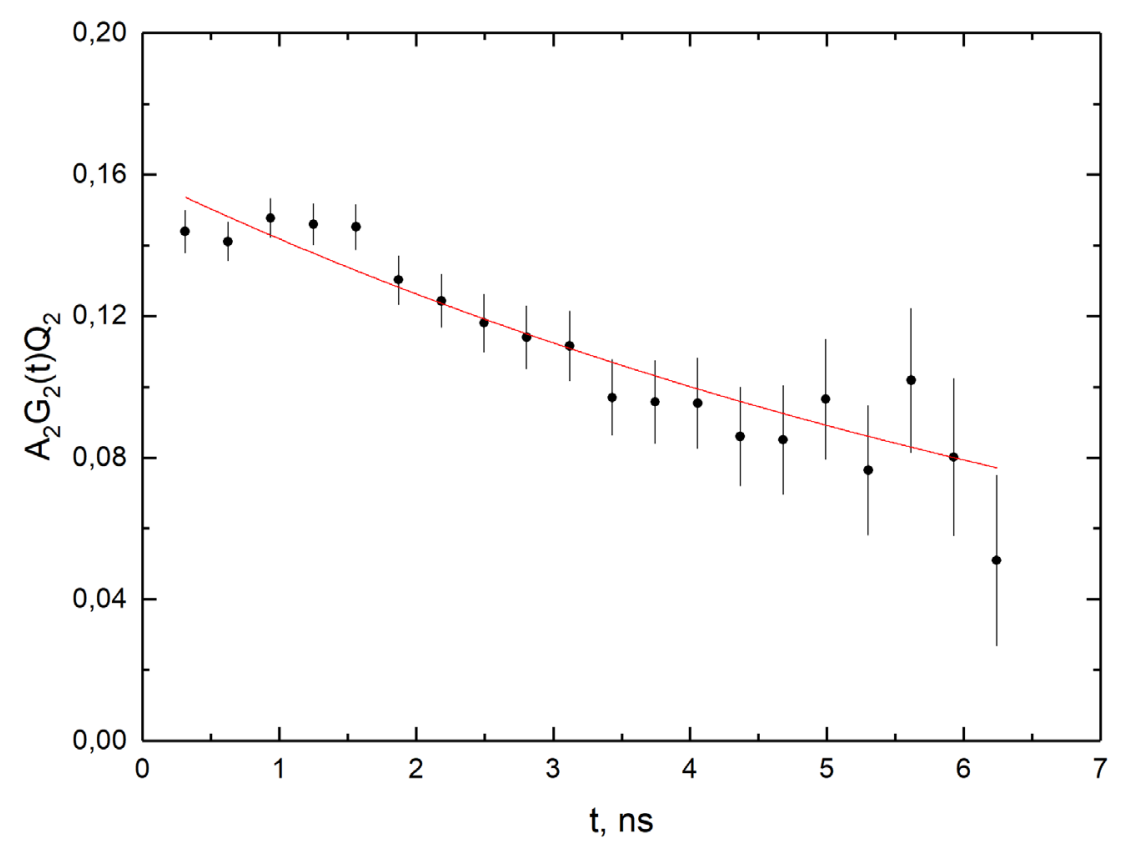

Figure S8. TDPAC spectra for ${ }^{154} \mathrm{Eu}$ at $\mathrm{C}(\mathrm{DTPA})=10^{-4} \mathrm{M}$, ionic strength $\mathrm{I}=0.5\left(\mathrm{HClO}_{4}\right.$, $\mathrm{NaClO}_{4}, \mathrm{NaOH}$ ) with $\mathrm{pH}=6.9$. 


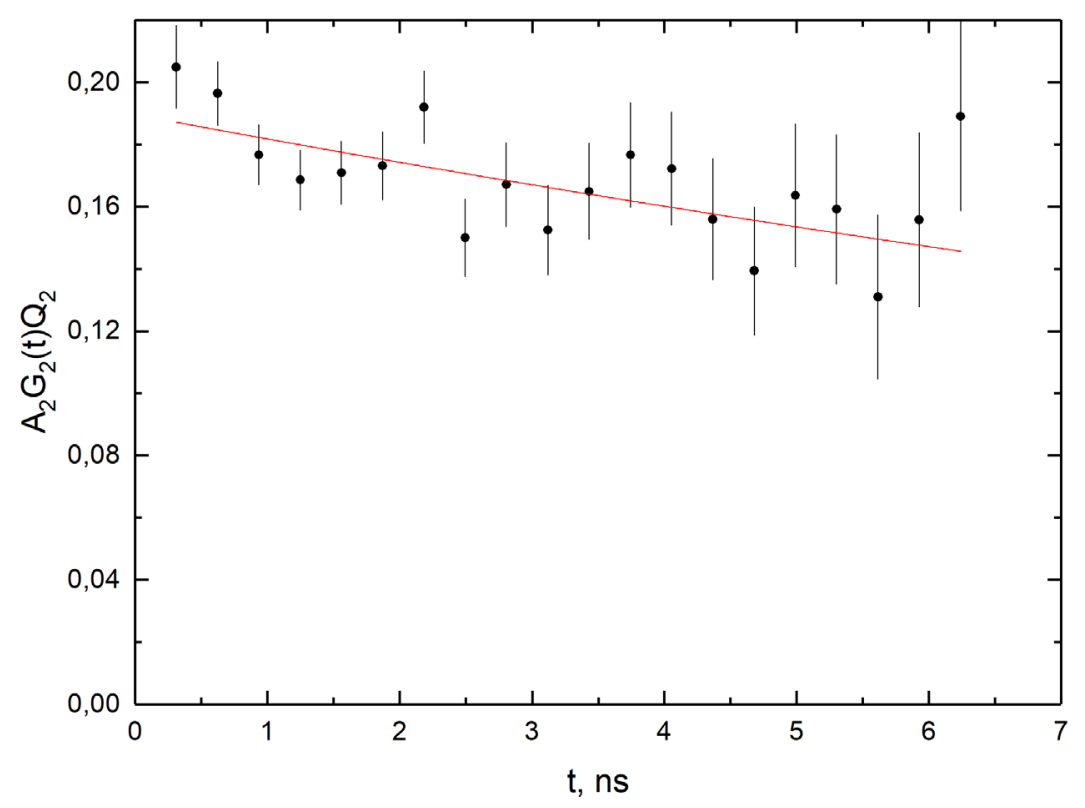

Figure S9. TDPAC spectra for ${ }^{152} \mathrm{Eu}$ at $\mathrm{C}(\mathrm{DTPA})=10^{-4} \mathrm{M}$, ionic strength $\mathrm{I}=0.5\left(\mathrm{HClO}_{4}\right.$, $\left.\mathrm{NaClO}_{4}, \mathrm{NaOH}\right)$ with $\mathrm{pH}=0.9$.

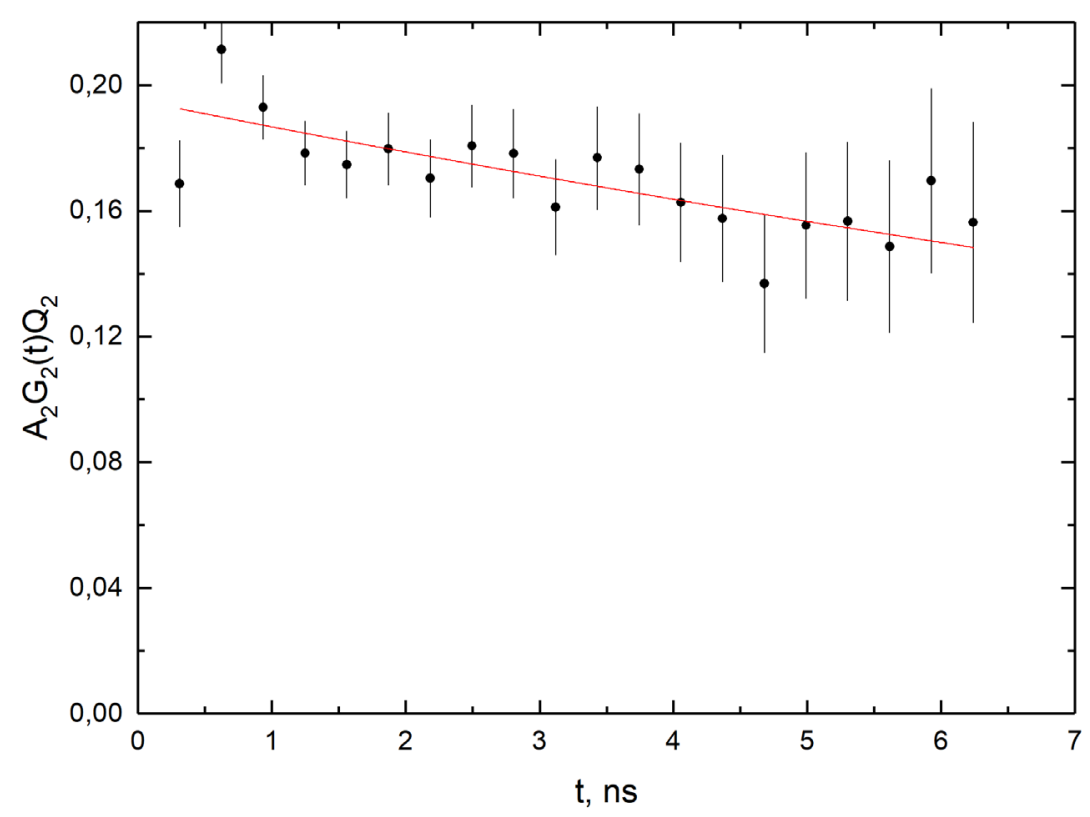

Figure S10. TDPAC spectra for ${ }^{152} \mathrm{Eu}$ at $\mathrm{C}(\mathrm{DTPA})=10^{-4} \mathrm{M}$, ionic strength $\mathrm{I}=0.5\left(\mathrm{HClO}_{4}\right.$, $\mathrm{NaClO}_{4}, \mathrm{NaOH}$ ) with $\mathrm{pH}=1.92$. 


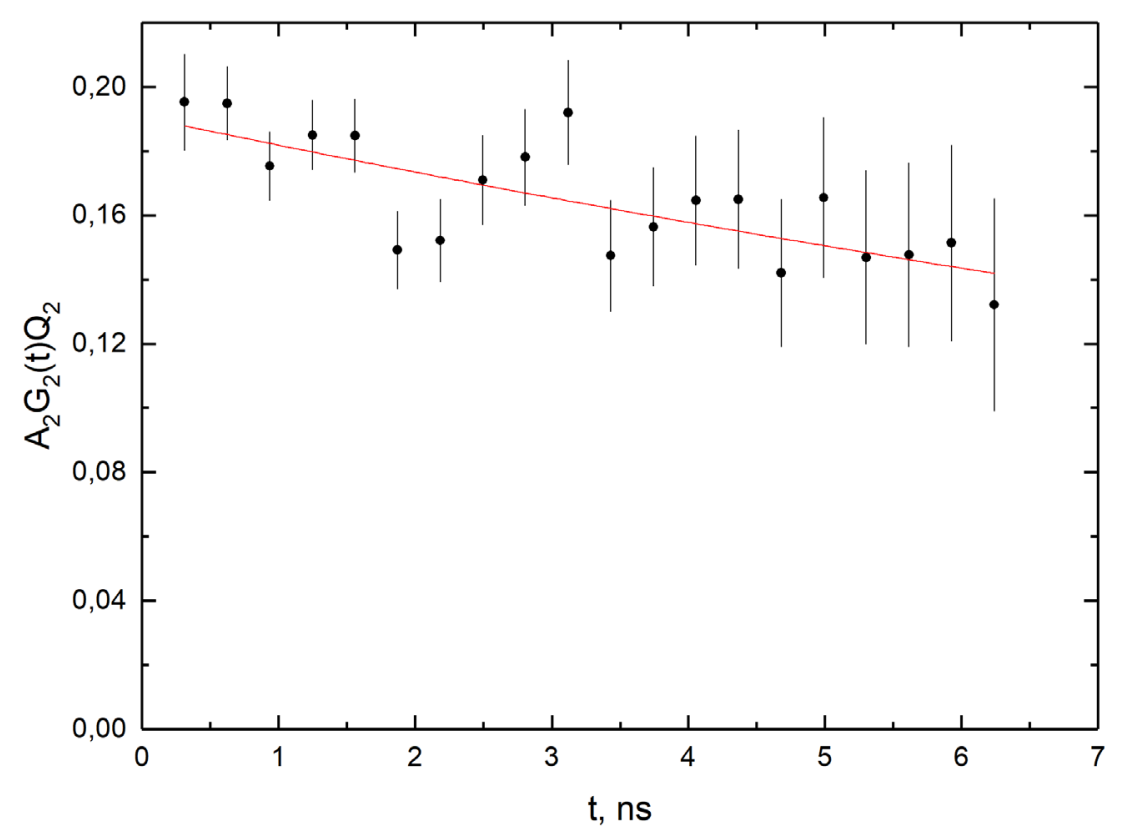

Figure S11. TDPAC spectra for ${ }^{152} \mathrm{Eu}$ at $\mathrm{C}(\mathrm{DTPA})=10^{-4} \mathrm{M}$, ionic strength $\mathrm{I}=0.5\left(\mathrm{HClO}_{4}\right.$, $\left.\mathrm{NaClO}_{4}, \mathrm{NaOH}\right)$ with $\mathrm{pH}=5.22$.

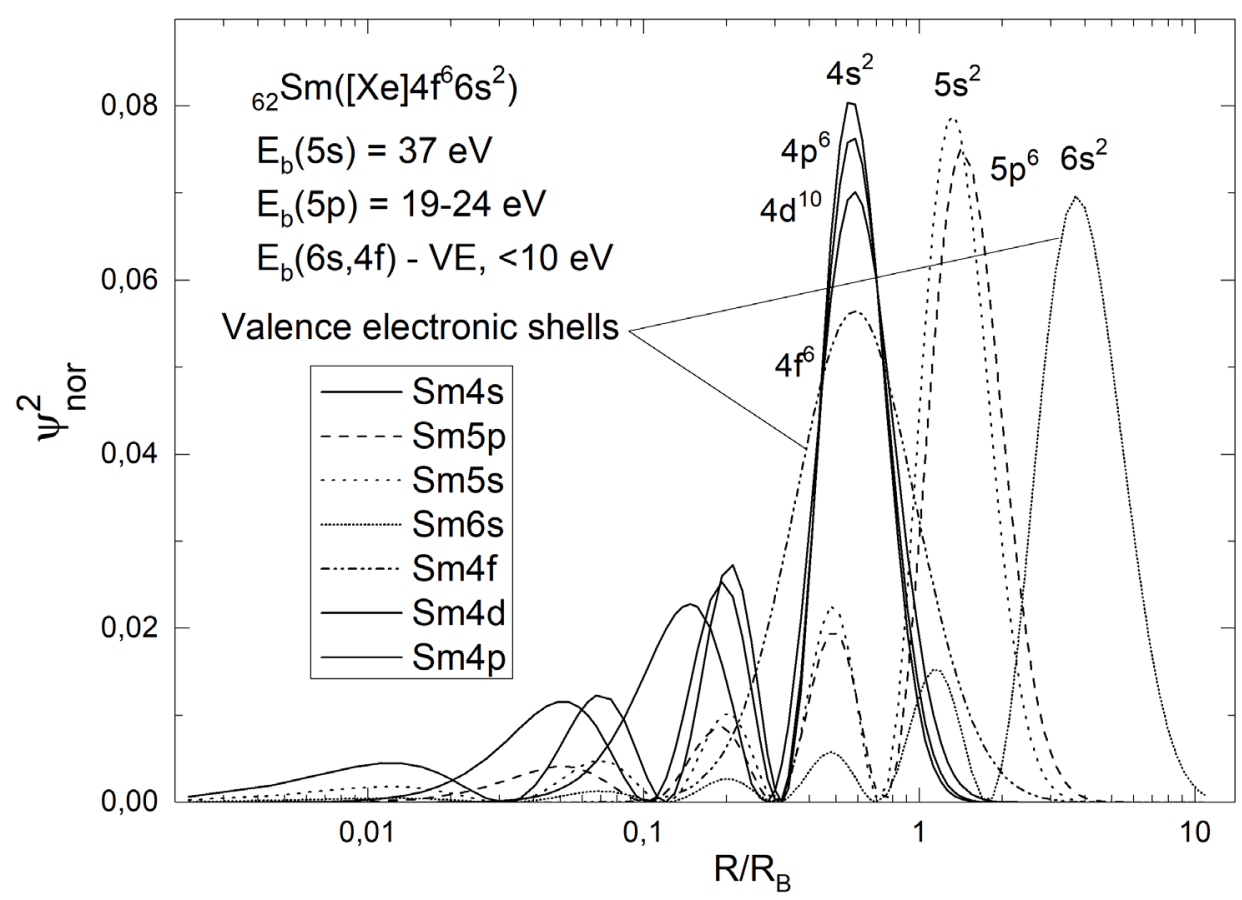

Figure S12. Charge distribution $\left(\psi^{2}\right)$ in electronic states of Sm free atom versus R distance from the nucleus in units of Bohr radius $\mathrm{R}_{\mathrm{B}}$. These data were extracted from ${ }^{1}$. 


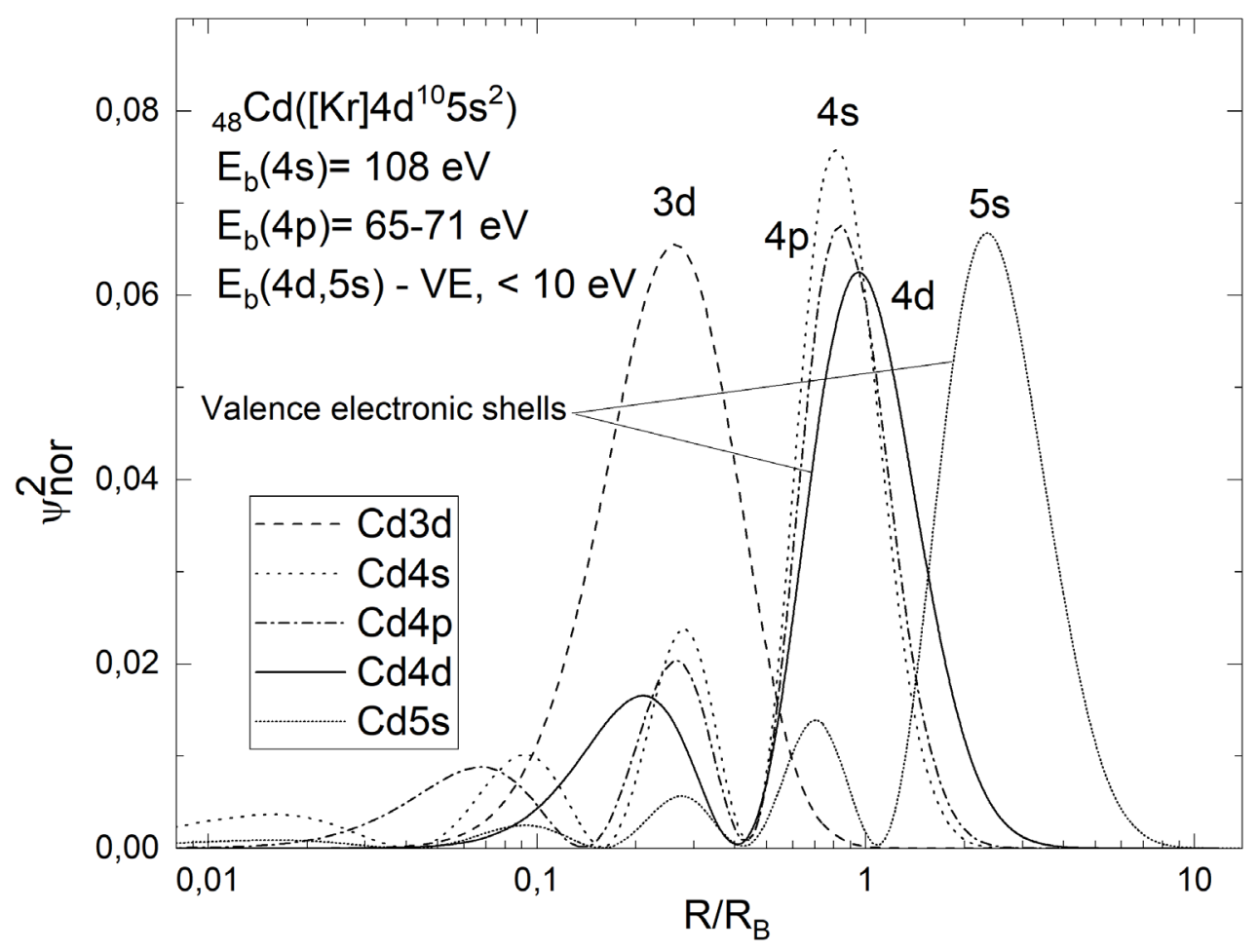

Figure S13. Charge distribution $\left(\psi^{2}\right)$ in electronic states of $\mathrm{Cd}$ free atom versus $\mathrm{R}$ distance from the nucleus in units of Bohr radius $\mathrm{R}_{\mathrm{B}}$. These data were extracted from ${ }^{1}$.

(1) Herman, F.; Skillman, S. Atomic Structure Calculations; Prentice-Hall Inc.: Englewood Cliffs, 1963. 